\title{
Distribution of Sterigmatocystin-producing Aspergilli in Japan
}

\author{
Naoki Kobayashi ${ }^{1}$, Atsutaka Kubosaki ${ }^{2}$, Yumi Takahashi ${ }^{3}$, Miyuki Yanai ${ }^{4}$, \\ Rumi Konuma ${ }^{5}$, Satomi Uehara ${ }^{3}$, Takashi Chiba ${ }^{3}$, Maiko Watanabe ${ }^{2}$, \\ Jun Terajima ${ }^{2, \dagger}$, and Yoshiko Sugita-Konishi ${ }^{1}$ \\ ${ }^{1}$ Department of Food and Life Sciences, Azabu University, 1-17-71 Fuchinobe Chuo-ku, Sagamihara, Kanagawa \\ 252-5201, Japan \\ ${ }^{2}$ National Institute of Health Sciences, 3-25-26 Tonomachi, Kawasaki-ku, Kawasaki, Kanagawa 210-9501, Japan \\ ${ }^{3}$ Tokyo Metropolitan Institute of Public Health, 3-24-1 Hyakunin-cho, Shinjuku-ku, Tokyo 169-0073, Japan \\ 4Japan Food Research Laboratories, Tama Laboratory, 6-11-10 Nagayama, Tama-shi, Tokyo 206-0025, Japan \\ ${ }^{5}$ Tokyo Metropolitan Industrial Technology Research Institute, 2-4-10 Aomi, Koto-ku, Tokyo 135-0064, Japan
}

\begin{abstract}
Sterigmatocystin is a genotoxic and hepatocarcinogenic mycotoxin that contaminates foods and environments worldwide. Sterigmatocystin is produced as a precursor to aflatoxin B1 or as an end product by certain Aspergilli. Aspergillus section Versicolores is one of the major sections including sterigmatocystin-producing species and is thus a potential health and environmental hazard. Recently, the taxonomy of this section was revised and classified into 14 species on the basis of molecular phylogenetic analysis. However, investigation of the distribution and sterigmatocystin production of each species has been limited; in particular, its distribution in foods has been scarcely reported. In this study, we collected isolates of Aspergillus section Versicolores from various foods and environments in Japan and investigated their distribution and sterigmatocystin production. The isolates were classified into nine species or species groups, which revealed that $A$. creber, A. puulaauensis/tennesseensis and A. sydowii are the main species/species groups in Japan. In addition, A. versicolor sensu stricto was detected with some frequency, specifically in foods. Furthermore, the two species A. creber and A. versicolor sensu stricto frequently produced sterigmatocystin. It is therefore important for food safety to intensively monitor these two species and distinguish them from other species, especially A. sydowii, which is not considered to produce sterigmatocystin.
\end{abstract}

Key words: sterigmatocystin, Aspergillus section Versicolores, fungi, distribution

\section{Introduction}

Sterigmatocystin (STC) is a toxic polyketide that is a precursor to aflatoxin B1 (AFB1), which is one of the most carcinogenic toxins in nature ${ }^{1,2)}$. The structure of STC is closely related to AFB1, but the lethal potency of STC is about $1 / 10$ that of $\mathrm{AFB1}^{3)}$, and the hepatocarcinogenicity is one or two orders of magnitude less than AFB1 as assessed in rats ${ }^{4}$. STC has been categorized as a $2 \mathrm{~B}$ carcinogen by the International Agency for Research on $\mathrm{Cancer}^{5}$ ). Furthermore, genotoxicity and carcinogenicity data were recently compiled by the European Food Safety Agency $(\mathrm{EFSA})^{6}$ demonstrating that STC induces chromosomal damage both in vitro and in vivo and tumorigenicity was observed after

Received: 2 February 2018; Accepted: 2 April 2018; Published online: 7 June 2018

Corresponding author: Prof. Yoshiko Sugita-Konishi, Department of Food and Life Sciences, Azabu University, 1-17-71 Fuchinobe

Chuo-ku, Sagamihara, Kanagawa 252-5201, Japan (y-konishi@azabu-u.ac.jp)

${ }^{\dagger}$ Current address: Iwate University, 3-18-8 Ueda, Morioka, 020-8550, Japan.

The contents of this article reflect solely the view of the author(s).

This paper was presented at the 12th International Symposium "Toxins, Pathogens, and Foods: Challenges and Opportunities for Public Health" organized by the Toxic Microorganisms Panel of the United States-Japan Cooperative Program on Development \& Utilization of Natural Resources.

Abbreviations: AFB1: aflatoxin B1, EFSA: European Food Safety Agency, STC: sterigmatocystin, TLC: thin-layer chromatography 
administration of STC in several animals.

STC contamination has been found in wheat, maize, barley, coffee beans, pistachio nuts and animal feed ${ }^{2,7,8)}$. STC has also been detected in various environmental samples such as carpet dust, dwelling materials within damp buildings and indoor air ${ }^{9,10)}$. Although STC is produced as a precursor to AFB1 in some Aspergillus species such as A. flavus and A. parasiticus, it is the end product in several species, such as A. nidulans, A. versicolor, A. aurolatus, Chaetomium spp., Emericilla spp., and Penicillium inflatum ${ }^{6,11)}$.

Aspergillus section Versicolores includes the major STC producers, which have been reported to be potential environmental and health hazards ${ }^{11-13)}$. The taxonomy of this section was recently revised, which increased the number of species from four to $14^{14)}$. The production of STC by each species in section Versicolores except for $A$. sydowii has been tested on two liquid media ${ }^{15}$ and revealed that 11 produce STC, namely $A$. amoenus, $A$. creber, $A$. cvjetkovicii, A. fructus, $A$. jensenii, A. protuberus, A. puulaauensis, A. subversicolor, $A$. tennesseensis, $A$. versicolor and $A$. venenatus. Two species (A. austroafricanus and A. tabacinus) were found not to produce STC. Sterigmatocystin production of $A$. sydowii was not tested because this species is generally regarded as a non-STC producer ${ }^{16)}$. However, the number of strains tested was limited; in particular, only one strain of $A$. austroafricanus and two strains of $A$. tabacinus were tested, and no STC production was reported. Thus, it is not certain whether these two species are indeed non-STC producers. Furthermore, most of the investigated strains were isolated from environmental or clinical samples, although many strains of Aspergillus section Versicolores were isolated from foods and feeds before the taxonomy was revised ${ }^{14,15)}$.

In this study, we performed molecular characterization of the isolates that had been morphologically associated with Aspergillus section Versicolores from various foods and environments in Japan, and also tested them for STC production.

\section{Materials and Methods}

\section{2-1. Strains}

The 60 strains used in this study are detailed in Table 1. Thirty strains were isolated from foods, and 30 were isolated from various environmental sources in regions of Japan such as Tokyo, Miyagi and Kumamoto prefectures. All strains were stored on potato dextrose agar (Eiken, Tokyo, Japan) slant medium at $25^{\circ} \mathrm{C}$.

\section{2-2. DNA Extraction and Sequencing}

Conidia from slant cultures were inoculated into $1 \mathrm{~mL}$ potato dextrose broth medium (Difco Laboratories, Franklin Lakes, NJ, USA) in a microfuge tube and incubated at $25^{\circ} \mathrm{C}$ for 3 days. The mycelia were clumped by centrifugation at $18,000 \times g$ for $10 \mathrm{~min}$. Genomic DNA from each strain was extracted using either the sodium dodecyl sulfate method $^{17)}$ or a DNeasy Plant Mini Kit (QIAGEN, Hilden, Germany). Partial sequences of the $\beta$-tubulin gene (tub2) were selected as the region for molecular phylogenetic analysis. For amplification and sequencing, primers Bt2a (5'-GGTAACCAAATCGGTGCTGCTTTC-3') and Bt2b (5'-ACCCTCAGTGTA GTGACCCTTGGC-3') were used as described ${ }^{18)}$. Amplification reactions were carried out in a thermal cycler (LifeECO; Bioer Technology, Hangzhou, China) with Ex Taq (TaKaRa Bio, Shiga, Japan). The PCR program for the amplification of $t u b 2$ consisted of an initial denaturing step at $95^{\circ} \mathrm{C}$ for $3 \mathrm{~min}, 35$ amplification cycles $\left(95^{\circ} \mathrm{C}\right.$ for $30 \mathrm{sec}, 60^{\circ} \mathrm{C}$ for $45 \mathrm{sec}, 72^{\circ} \mathrm{C}$ for $\left.30 \mathrm{sec}\right)$ and an additional extending step at $72^{\circ} \mathrm{C}$ for $2 \mathrm{~min}$. The amplified fragments were sequenced using an ABI 3700 Analyzer and the BigDye Terminator Sequencing reagent (Thermo Fisher Scientific, Waltham, MA, USA).

\section{2-3. Identification of Species}

Isolate identities were confirmed or refuted based on the tub2 genomic region. Twenty-six sequence datasets of Aspergillus section Versicolores were downloaded from GenBank and used as reference data for our phylogenetic inference. A. asperescens and A. multicolor were used as out-group taxa, and their tub2 sequences were also downloaded from GenBank. The sequences were automatically aligned using MEGA6 ${ }^{19)}$, and the alignments were manually adjusted. A phylogenetic tree was constructed with the neighbor-joining method by the maximum composite likelihood model using MEGA6. The stability of clades was assessed with 1,000 bootstrap replications.

\section{2-4. STC Analysis by Thin-layer Chromatogra- phy (TLC)}

One-dimensional TLC was carried out as previously described $^{20)}$ with some modifications. In brief, isolates were cultured on potato dextrose agar at $25^{\circ} \mathrm{C}$ for 14 days. Using a $1 \mathrm{~mL}$ pipette tip, agar plugs were removed from cultures and placed into $1.5 \mathrm{~mL}$ sample tubes. The plugs were treated with methanol-chloroform [1:2 (vol/vol)], and an aliquot of each extract was directly transferred onto a TLC plate (Silica gel 60, Merck, Darmstadt, Germany) that had been previously activated for $15 \mathrm{~min}$ at $120^{\circ} \mathrm{C}$. The plates were developed in methanol-chloroform [2:98 (vol/vol)] and dried. Metabolites were visualized under UV light at $366 \mathrm{~nm}$ and compared to a STC standard (Major Chemicals Ltd, Rehovot, Israel). Me- 
Table 1. Aspergillus strains used in this study

\begin{tabular}{|c|c|c|c|c|}
\hline Species/Species group & Strain & Origin & STC production ${ }^{\mathrm{a}}$ & Accession No. ${ }^{b}$ \\
\hline A. amoenus & NIHS6481 & House dust & - & LC367586 \\
\hline \multirow[t]{12}{*}{ A. creber } & FSSN0002 & Canned Truffle & - & LC367551 \\
\hline & NIHS0585 & Hair of cattle & - & LC367554 \\
\hline & NIHS0586 & House dust & + & LC367555 \\
\hline & NIHS1302 & Grape & + & LC367559 \\
\hline & NIHS2991 & Indoor air & + & LC367561 \\
\hline & NIHS3057 & Indoor air & + & LC367562 \\
\hline & NIHS4459 & Environment & + & LC367571 \\
\hline & NIHS6467 & House dust & + & LC367579 \\
\hline & NIHS6718 & Dust of bed & + & LC367592 \\
\hline & NIHS6806 & Cocoa powder & - & LC367599 \\
\hline & Tokyo-AV-54 & Olive oil & + & LC367605 \\
\hline & Tokyo-AV-63 & Barley tea & - & LC367610 \\
\hline \multirow[t]{4}{*}{ A. jensenii } & NIHS3160 & Indoor air & + & LC367563 \\
\hline & NIHS3253 & Indoor wall & - & LC367565 \\
\hline & NIHS6501 & House dust & - & LC367591 \\
\hline & Tokyo-AV-19 & Pilled millet & + & LC367604 \\
\hline \multirow[t]{2}{*}{ A. protuberus } & NIHS6474 & House dust & - & LC367582 \\
\hline & NIHS6482 & House dust & - & LC367587 \\
\hline \multirow[t]{11}{*}{ A. puulaauensis/A. tennesseensis } & NIHS0581 & Vinyl-coated cloth & - & LC367553 \\
\hline & NIHS6259 & Polished rice & - & LC367572 \\
\hline & NIHS6262 & Polished rice & + & $\mathrm{LC} 367574$ \\
\hline & NIHS6270 & Polished rice & + & LC367577 \\
\hline & NIHS6466 & House dust & - & LC367578 \\
\hline & NIHS6480 & House dust & - & LC367585 \\
\hline & NIHS6805 & Lemon juice & - & LC367597 \\
\hline & NIHS6807 & Cocoa powder & - & LC367598 \\
\hline & Tokyo-AV-15 & Soy beans & + & LC367602 \\
\hline & Tokyo-AV-16 & Jasmine tea leaf & + & LC367603 \\
\hline & Tokyo-AV-55 & Turmeric & - & LC367606 \\
\hline
\end{tabular}


Table 1. Aspergillus strains used in this study (continued)

\begin{tabular}{|c|c|c|c|c|}
\hline Species/Species group & Strain & Origin & STC production ${ }^{\mathrm{a}}$ & Accession No. ${ }^{b}$ \\
\hline \multirow[t]{22}{*}{ A. sydowii } & NIHS0733 & Fennel & - & LC367557 \\
\hline & NIHS1013 & Agar weed & - & LC367558 \\
\hline & NIHS3261 & Outdoor air & - & LC367566 \\
\hline & NIHS3404 & Outdoor air & - & LC367567 \\
\hline & NIHS3941 & Indoor air & - & LC367568 \\
\hline & NIHS4021 & Indoor air & - & LC367569 \\
\hline & NIHS4095 & Indoor wall & - & LC367570 \\
\hline & NIHS6261 & Polished rice & - & LC367573 \\
\hline & NIHS6265 & Polished rice & - & LC367576 \\
\hline & NIHS6469 & House dust & - & LC367580 \\
\hline & NIHS6472 & House dust & - & LC367581 \\
\hline & NIHS6478 & House dust & - & LC367583 \\
\hline & NIHS6479 & House dust & - & LC367584 \\
\hline & NIHS6497 & House dust & - & LC367588 \\
\hline & NIHS6498 & House dust & - & LC367589 \\
\hline & NIHS6499 & House dust & - & LC367590 \\
\hline & NIHS6801 & Peanuts & - & LC367593 \\
\hline & NIHS6802 & Peanuts & - & LC367594 \\
\hline & NIHS6803 & Beverage & - & LC367595 \\
\hline & NIHS6804 & Coconut oil & - & LC367596 \\
\hline & Tokyo-AV-58 & Dried fish & - & LC367608 \\
\hline & Tokyo-AV-60 & Roasted green tea & - & LC367609 \\
\hline A. tabacinus & NIHS0587 & Cellophane & - & LC367556 \\
\hline \multirow[t]{3}{*}{ A. venenatus } & NIHS3237 & Indoor wall & - & LC367564 \\
\hline & NIHS6808 & Soy beans & + & LC367600 \\
\hline & NS270272 & Rice grain & - & LC367601 \\
\hline \multirow[t]{4}{*}{ A. versicolor sensu stricto } & NIHS0093 & Rice grain & + & LC367552 \\
\hline & NIHS2500 & Rice grain & + & LC367560 \\
\hline & NIHS6264 & Polished rice & + & LC367575 \\
\hline & Tokyo-AV-57 & Noodle & - & LC367607 \\
\hline
\end{tabular}

a Sterigmatocystin production: + is producing, - is non-producing

${ }^{b}$ GenBank accession number for partial sequences of $\beta$-tubulin genes 
tabolites demonstrating similar $\mathrm{Rf}$ and color to the standard were regarded as STC.

\section{Results}

\section{3-1. Identification of Species}

To assign isolates into the 14 species in Aspergillus section Versicolores described by Jurjevic et al. ${ }^{14)}$, the isolates were subjected to molecular phylogenetic analysis based on tub2 with the 26 sequences of Aspergillus section Versicolores strains used in the previous study ${ }^{14)}$. We obtained the neighbor-joining tree using 88 sequences (Fig. 1). Each reference species formed a monophyletic clade, and seven of the clades were supported with high bootstrap values (BP, $>80 \%$ ). Of the 60 strains isolated in this study, 22 were identified as $A$. sydowii and 12 as $A$. creber. The support values for both clades were very high ( $>99 \%$ BP). We also identified the following strains with high bootstrap support, i.e., $>80 \%$ BP: four strains of $A$. versicolor sensu stricto, three strains of $A$. venenatus, two strains of $A$. protuberus, one strain of A. amoenus, and one strain of A. tabacinus. Among the 15 remaining strains, four strains formed a monophyletic clade with $A$. jensenii (69\% BP) and 11 formed a monophyletic clade with $A$. puulaauensis and A. tennesseensis (66\% BP).

\section{3-2. Distribution of Species}

Among the 30 strains isolated from food, there were 10 strains of $A$. sydowii, eight in the species group of $A$. puulaauensis and A. tennesseensis, five of $A$. creber, four of $A$. versicolor sensu stricto, two of $A$. venenatus, and one of $A$. jensenii (Table 1). Among the 30 strains isolated from the environment, there were 12 strains of $A$. sydowii, seven of $A$. creber, three of $A$. jensenii, three in the species group of $A$. puulaauensis and $A$. tennesseensis, two of $A$. protuberus, one of $A$. amoenus, one of $A$. tabacinus and one of $A$. venenatus.

\section{3-3. STC Production}

Of the 60 isolates tested, $18(30.0 \%)$ produced STC as assessed with TLC. Specifically, the following isolates produced STC: 8 of 12 isolates (66.7\%) of $A$. creber, 4 of 11 isolates (36.4\%) in the species group of $A$. puulaauensis and $A$. tennesseensis, 3 of 4 isolates (75\%) of $A$. versicolor sensu stricto, 2 of 4 isolates (50.0\%) of $A$. jensenii, and 1 of 3 isolates (33.3\%) of $A$. venenatus. The isolates of the other six species did not produce STC. In particular, none of the 22 isolates of $A$. sydowii produced STC.

\section{Discussion}

Sixty strains, morphologically identified as belonging to
Aspergillus section Versicolores, were isolated from various foods and environments in Japan. Using molecular phylogenetic inference, the strains were partitioned into nine species or species groups. Most of the isolates were identified as $A$. sydowii (22 isolates), followed by $A$. creber (12 isolates) and the species group of $A$. puulaauensis and A. tennesseensis (11 isolates) (Table 1 and Fig. 1). These three species or species groups made up two-thirds of the isolates, indicating that they likely comprise the majority species of Aspergillus section Versicolores in Japan. This is the first report of the distribution in Japan of species belonging to Aspergillus section Versicolores. In previous studies carried out subsequent to the reclassification of Aspergillus section Versicolores ${ }^{13,14)}$, $A$. creber was the species most commonly isolated, followed by $A$. jenseniii and $A$. protuberus. Although $A$. sydowii cannot be compared with previous results because it was excluded from the analyses, the frequency of $A$. creber in the present study agrees with that reported previously. Our findings, however, differ from previous results in that $A$. jensenii and $A$. protuberus were not frequently isolated in our study. There may have been fewer isolates of $A$. jensenii and $A$. protuberus in our study because different sources were sampled. Although the previous studies mainly used isolates from the environment or clinical specimens, we collected half of the isolates from food. Thus, $A$. jensenii and $A$. protuberus might be abundantly distributed in the environment but rarely in food. On the other hand, A. creber was detected in our study as frequently as in previous studies. Although $A$. creber has not previously been isolated from food, we isolated a similar number of $A$. creber strains from food and environmental samples in our study. Thus, it appears that $A$. creber is frequently distributed in both the environment and food. Furthermore, A. versicolor sensu stricto was isolated only from foods in both previous studies and our study, indicating that this species is mainly distributed in food. Most of the isolates identified as $A$. versicolor sensu lato from food and feed, prior to reclassification of the species in Aspergillus section Versicolores, might be $A$. versicolor sensu stricto.

Aspergillus section Versicolores is comprised of a major group of STC producers that have been reported to be potential environmental and health hazards ${ }^{11,12}$. After reclassification of this section, STC-producing strains were detected in most species of this section. Our finding that none of the 22 A. sydowii isolates produced STC supports the belief that this is a non-STC species, but this does not mean that an STCproducing strain of $A$. sydowii does not exist. Therefore, any sampling of $A$. sydowii should be subjected to STC testing. Because $A$. versicolor sensu stricto and $A$. creber frequently produce STC, and because these two species were frequently isolated from food, they are likely to be the main cause of 


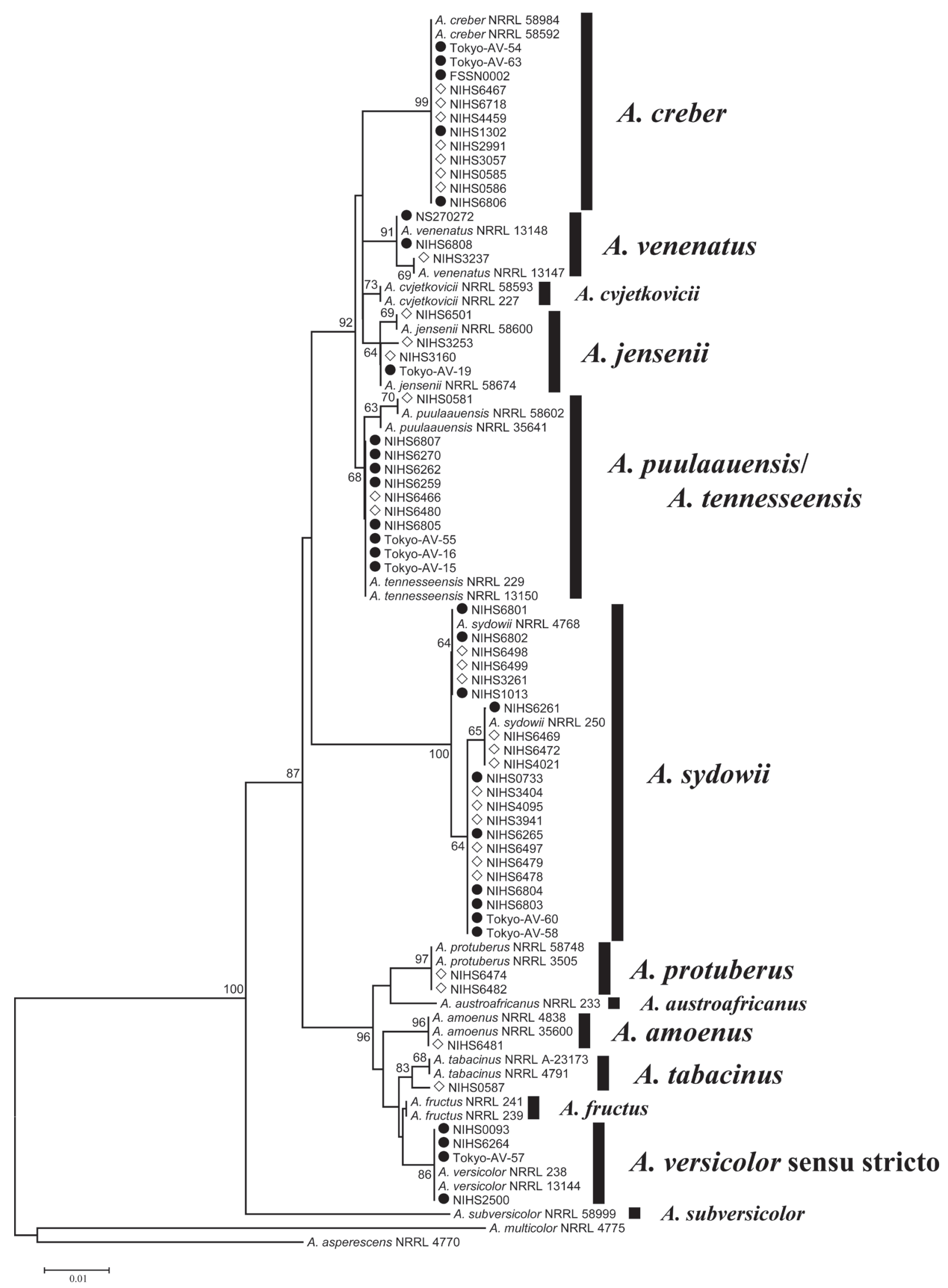

Fig. 1. Phylogenetic tree based on partial sequences of $\beta$-tubulin. The tree was generated by the neighbor-joining method using 1,000 bootstrap replicates. Only the bootstrap values above $60 \%$ are indicated at the branches. The tree was rooted with outgroup taxa $A$. asperescens and A. multicolor. The strains marked by black circles were isolated from foods; the strains marked by white diamonds were isolated from environmental samples; the sequences of the unmarked strains were downloaded from GenBank. 
STC contamination in food and feed in Japan. Therefore, it is important for purposes of food safety to intensively monitor foods for these two particular species. Because we examined STC production only in 2-week cultures at $25^{\circ} \mathrm{C}$ on potato dextrose agar, STC production of each species from Aspergillus section Versicolores should be examined under additional conditions. Further understanding of the distribution and STC production by strains of Aspergillus section Versicolores isolated from additional locations is needed and will lead to improved food safety.

\section{Acknowledgments}

This work was supported by the Health and Labor Sciences Research Grants (Research on Food Safety, H28-shokuhin-ippan-004) from the Ministry of Health, Labor and Welfare of Japan.

\section{Conflict of interest}

The authors have no conflict of interest.

\section{References}

1. Terao K. Sterigmatocystin-a masked potent carcinogenic mycotoxin. J Toxicol Toxin Rev. 1983; 2: 77-110.

2. Veršilovskis A, De Saeger S. Sterigmatocystin: occurrence in foodstuffs and analytical methods-an overview. Mol Nutr Food Res. 2010; 54: 136-147. PMID:19998385, doi:10.1002/ mnfr.200900345

3. Purchase IFH, Van der Watt JJ. Acute toxicity of sterigmatocystin to rats. Food Cosmet Toxicol. 1969; 7: 135-139. PMID:5822868, doi:10.1016/S0015-6264(69)80295-6

4. Purchase IFH, van der Watt JJ. Carcinogenicity of sterigmatocystin. Food Cosmet Toxicol. 1970; 8: 289-295. PMID:4318199, doi:10.1016/S0015-6264(70)80004-9

5. IARC. Overall evaluations of carcinogenicity: an updating of IARC Monographs Volumes 1 to 42. IARC Monogr Eval Carcinog Risk Chem Hum. 1987; 1-42: 64.

6. Böhm J, De Saeger S, Edler L. Scientific Opinion on the risk for public and animal health related to the presence of sterigmatocystin in food and feed. EFSA J. 2013; 11: 1-81.

7. Mol HGJ, Pietri A, Macdonald SJ, Anagnostopoulos C. Survey on sterigmatocystin in food - EFSA supporting publication. EFSA Support Publ. 2015; 12: 1-56.

8. Pitt JI, Hocking AD. Fungi and food spoilage. Fungi and Food Spoilage. 2009: doi: doi:10.1007/978-0-387-92207-2.
9. Bloom E, Nyman E, Must A, Pehrson C, Larsson L. Molds and mycotoxins in indoor environments--a survey in waterdamaged buildings. J Occup Environ Hyg. 2009; 6: 671-678. PMID:19757292, doi:10.1080/15459620903252053

10. Tuomi T, Reijula K, Johnsson T, et al. Mycotoxins in crude building materials from water-damaged buildings. Appl Environ Microbiol. 2000; 66: 1899-1904. PMID:10788357, doi:10.1128/AEM.66.5.1899-1904.2000

11. Rank C, Nielsen KF, Larsen TO, Varga J, Samson RA, Frisvad JC. Distribution of sterigmatocystin in filamentous fungi. Fungal Biol. 2011; 115: 406-420. PMID:21530923, doi:10.1016/j.funbio.2011.02.013

12. Bellanger AP, Reboux G, Roussel S, et al. Indoor fungal contamination of moisture-damaged and allergic patient housing analysed using real-time PCR. Lett Appl Microbiol. 2009; 49: 260-266. PMID:19486284, doi:10.1111/j.1472765X.2009.02653.X

13. Despot DJ, Kocsubé S, Bencsik O, et al. Species diversity and cytotoxic potency of airborne sterigmatocystin-producing Aspergilli from the section Versicolores. Sci Total Environ. 2016; 562: 296-304. PMID:27100010, doi:10.1016/j.scitotenv.2016.03.183

14. Jurjevic Z, Peterson SW, Horn BW. Aspergillus section Versicolores : nine new species and multilocus dNA sequence based phylogeny. IMA Fungus. 2012; 3: 59-79. PMID:23155501, doi:10.5598/imafungus.2012.03.01.07

15. Jurjević Ž, Peterson SW, Solfrizzo M, Peraica M. Sterigmatocystin production by nine newly described Aspergillus species in section Versicolores grown on two different media. Mycotoxin Res. 2013; 29: 141-145. PMID:23417508, doi:10.1007/s12550-013-0160-4

16. Frisvad JC, Thrane U. Chapter 4 Mycotoxin production by food-borne fungi. Introduction to food-borne fungi. Somson RA, Hoekstra ES, Frisvad JC, Filtenborg O, eds. Baarn, The Netherlands: Centraalbureau voor Schimmelcultures; 1995: 251-260

17. Watanabe M, Lee K, Goto K, Kumagai S, Sugita-Konishi Y, Hara-Kudo Y. Rapid and effective DNA extraction method with bead grinding for a large amount of fungal DNA. J Food Prot. 2010; 73: 1077-1084. PMID:20537263, doi:10.4315/0362-028X-73.6.1077

18. Glass NL, Donaldson GC. Development of primer sets designed for use with the PCR to amplify conserved genes from filamentous ascomycetes. Appl Environ Microbiol. 1995; 61: 1323-1330. PMID:7747954

19. Tamura K, Stecher G, Peterson D, Filipski A, Kumar S. MEGA6: Molecular evolutionary genetics analysis version 6.0. Mol Biol Evol. 2013; 30: 2725-2729. PMID:24132122, doi:10.1093/molbev/mst197

20. Filtenborg O, Frisvad JC, Svendsen JA. Simple screening method for molds producing intracellular mycotoxins in pure cultures. Appl Environ Microbiol. 1983; 45: 581-585. PMID:6338829 